\title{
Recursive travel-time inversion: A tool for real-time seismic tomography
}

\author{
Masashi Ogiso $^{1 *}$, Kiyoshi Yomogida ${ }^{1}$, and Kei Katsumata ${ }^{2}$ \\ ${ }^{1}$ Division of Earth and Planetary Sciences, Graduate School of Science, Hokkaido University, Sapporo 060-0810, Japan \\ ${ }^{2}$ Institute of Seismology and Volcanology Graduate School of Science, Hokkaido University Sapporo 060-0810, Japan
}

(Received November, 16, 2004; Revised April 13, 2005; Accepted May 2, 2005)

\begin{abstract}
A new recursive inverse scheme is applied to a currently popular problem named seismic travel-time tomography, in order to enhance the efficiency and reliability in obtaining a new velocity model if a small number of new data are added to a large data set in the past. In comparison with conventional inverse schemes in seismic tomography, either least-squares or iterative types, this scheme does not require large amounts of matrix-type computations but utilizes the amount of modification in model parameters responsible for each new data set. We also introduce the computation of a collocation travel time (i.e., from a given station to every grid point) for the reference velocity model inverted by the data for all the past events, using a ray tracing scheme called the Huygens' method (Saito, 2001), suitable to computations prior to a new event. Combining the above information already stored with the recursive inverse scheme, we can obtain a new or updated velocity model immediately after a new event takes place, because a temporal interval between two events is usually very long in a given local area. Since the model is revised at each recursive step, we perform ray tracings with the updated reference model to get more accurate ray paths and travel times than the conventional inversion schemes that use all the ray tracings for the same reference model. We first showed the validity and stability of the proposed method with synthetic data. We then applied the new approach to the $P$-wave travel-time data recorded in the Hidaka, south-central Hokkaido, Japan, region, and compared our result with other previous results. Our result shares the overall feature with the previous ones. In addition, a new low-velocity zone is detected in the east of the Hidaka mountains at the depth of $10 \mathrm{~km}$, corresponding to the collision zone of two arcs, due to the use of the updated reference velocity model at each recursive step. We also confirmed that the order of data does not affect the final result, so that the present approach is shown as an appropriate tool for so-called real-time seismic tomography: a updated velocity model is immediately obtained at each time that a new event takes place, in order to monitor temporal variations of model parameters such as velocity structure on the real-time basis.
\end{abstract}

Key words: Seismic tomography, recursive inverse scheme, velocity structure, Hidaka mountains.

\section{Introduction}

Obtaining three-dimensional velocity structure by seismic travel-time data is one of the most active research fields in the present earthquake today, starting works such as Aki and Lee (1976) and Aki et al. (1977). The rapid development of both temporary and stationary seismic networks over the world has led to the flourish of this type of studies from global to extremely local problems (e.g., Nolet, 1987; Iyer and Hirahara, 1993). Three-dimensional velocity models are routinely obtained wherever a certain number of seismometers are installed, under a popular name of "Seismic Tomography". Its basic methodology consists of two parts: (1) ray tracing connecting each pair of source and receiver, and (2) solving an inverse problem with a large but sparse matrix. Both parts have been studied intensively, and there appear no rooms for improvement at least in theoretical points of view.

As the number of data increases rapidly, researchers are

*Present address: Osaka Aviation Weather Station, Japan Meteorological Agency, 3-371 Hotarugaike-Nishimachi, Toyonaka 560-0036, Japan.

Copy right(c) The Society of Geomagnetism and Earth, Planetary and Space Sciences (SGEPSS); The Seismological Society of Japan; The Volcanological Society of Japan; The Geodetic Society of Japan; The Japanese Society for Planetary Sciences; TERRAPUB trying to obtain results with very high spatial resolution. In order to fulfill this demand, one may introduce an accurate and complex velocity structure as the reference or initial model, and several new approaches have been proposed for computing ray paths and travel times in such complex media very accurately, for example, bending method (Zhao et al., 1992) and finite difference method (Vidale, 1990; Qin et al., 1992). The introduction of non-linear inverse schemes is another attempt to obtain a stable and accurate final result effectively, such as generic algorithm and simulated annealing, as summarized by Sen and Stoffa (1995).

One important aspect that has been investigated little yet in seismic tomography is the detection of temporal variations in velocity structure. If we had very accurate and spatially dense travel-time data available, slight temporal variations in subsurface velocity might be detected. In research fields other than earthquake seismology, this "realtime tomography" has been already explored, for example, for laser optics (e.g., Zvyagin et al., 2000; Ding et al., 2002) and medical treatment (e.g., Park et al., 2003). Real-time monitoring of spatial distributions of both water vapor in atmosphere and total electron content in ionosphere is now routinely conducted, using the GPS system (e.g., GPS Research Group, 2004). 
As far as the authors know, no serious studies on realtime tomography have been ever attempted in earthquake seismology. Any temporal variations in seismic velocity related to either earthquakes or volcanic activities have high potential for understanding their dynamic processes. One may obtain such a temporal variation with the conventional tomographic approach by taking the difference in results between two data sets, one for an early period and the other for a later period, as mentioned in other branches of science and engineering. A very subtle change in velocity structure in time has been recognized to be one reason why this approach has not been successful yet in earthquake seismology (e.g., Niu et al., 2003). Moreover, the above straightforward approach is neither suitable nor practical because of a very singular temporal pattern in earthquake occurrence in general, that is, virtually a small number of events in a normal period versus very concentrated occurrence in short time intervals associated with a major event. In other words, we routinely have plenty of "idle" time for new data to come while a new velocity distribution must be obtained immediately after a highly active period of seismicity starts. This singular character in real-time seismic tomography requires the introduction of a new tomographic approach, different from those developed before, as well as in other research fields.

In this study, we shall introduce a new inversion scheme suitable for the above special character in real-time seismic tomography, called recursive inverse scheme. Unlike the other inverse schemes, this scheme adopts an approach to modify the initial model sequentially at every step that a new data set becomes available. Before an active period of seismicity, we are supposed to have obtained a velocity model with the data acquired by that time. After new data become available, a new velocity model is obtained with the small amount of computations only for the new data, without taking into consideration the data that have been already inverted. We shall also introduce a ray-tracing scheme named the Huygens' method (Saito, 2001) by collocating source and station, so that the information of ray paths and their travel times from a given station to all the grid points in a medium has been computed and stored a priori. Combining these two new approaches, we can quickly obtain a new velocity model, putting real-time seismic tomography to practical use in future. We shall show the stability and accuracy of our approach with the data set in the Hidaka, Hokkaido, Japan, region, in comparison with the previous results obtained by conventional methods.

\section{Method}

Using the Fermat's principle, the travel-time residual $\delta T$ defined below is linearly related to the perturbation of slowness $\delta u$ ( $u \equiv 1 / v$ where $v$ is the velocity of $P$ or $S$ wave) along the ray path obtained for the reference velocity model up to the first order:

$$
\delta T \equiv T_{c a l c}-T_{o b s} \simeq \int_{p a t h}\left(u_{r e f}-u_{a c t}\right) d s \equiv \int_{p a t h} \delta u d s
$$

where $T_{\text {calc }}$ is the travel time calculated for the reference model, $T_{o b s}$ is the observed travel time, $u_{r e f}$ is the reference model of slowness, and $u_{\text {act }}$ is its actual distribution. In a practical inverse problem, we discretize the model space into small blocks or in a representation with the finite number of model parameters. For the $k$-th observation, $\delta T_{k}$, of the total of $N$ observations with the slowness perturbation of the $i$-th block, $\delta u_{i}$, Eq. (1) can be approximately expressed by

$$
\delta T_{k}=\sum_{i=1}^{M} s_{k i} \delta u_{i} \quad(k=1,2, \ldots, N)
$$

where $s_{k i}$ denotes the length of the $k$-th ray path in the $i$-th block of the total of $M$ blocks. We can write Eq. (2) in a matrix form as follows:

$$
\delta \mathbf{d}=\mathbf{G} \delta \mathbf{m}
$$

with the $N$-dimensional data vector $\delta \mathbf{d}$ with $\delta T_{k}$ as its $k$-th element, the $N \times M$ matrix $\mathbf{G}$ with $s_{k i}$ as its $k i$-th element, and the $M$-dimensional model vector $\delta \mathbf{m}$ with $\delta u_{i}$ as its $i$-th element.

Compared with conventional inversions in seismic traveltime tomography, we introduce two new approaches in this study, in order to meet the efficiency required by realtime seismic tomography: (a) ray tracing by the Huygens' method with the collocation of source and station, and (b) solving the matrix problem of Eq. (3) by a recursive inverse scheme.

Among a variety of ray-tracing methods that have been introduced in travel-time tomography in earthquake seismology (e.g., Iyer and Hirahara, 1993), we adopt the Huygens' method developed by Saito (2001). In this approach, we assign grid points in model space, then search the minimum in travel time among all the possible ray paths connecting a source and a given grid point, which modifies the conventional graph or network theory (e.g., Nishi, 2001) developed in other research fields to be suitable in earthquake and exploration seismology. A similar approach was also recently proposed by Rawlison and Sambridge (2004), investigating its efficiency and accuracy extensively. Although it can compute only the travel time and ray path of the first arrival phase at a given grid point (or a station), this approach is based on the computation of wave front so that ray paths and travel times can be obtained more accurately than the other methods based on the computation of ray paths, particularly for a medium with strong velocity gradient, as shown in the above studies.

In the application to real-time seismic tomography, this method is extremely suitable because its algorithm simultaneously computes ray paths and travel times for all the model or grid points from a given source point. Collocating source and stations, that is, assuming a source located at a given station, this method provides ray paths and travel times for all the grid points at once. If this procedure is conducted prior to a new event, we only need to pick up the ray path and travel time for the grid point where a new event is located (i.e., $s_{k i}$ and $\delta T_{k}$ in Eq. (2)). In other words, we do not compute ray paths and travel times after a new event occurs, unlike in the conventional seismic tomography.

Next, let us explain the recursive inverse scheme to solve the matrix system of Eq. (3). Among references in geo- 
physics, we can trace its original idea back to a review paper by Rodgers (1976). This method, called also Kalmanfilter formulation, has been used in some other branches of earth sciences, such as Global Positioning System (e.g., Hofmann-Wellenhof, 2001) and ocean acoustic tomography (e.g., Wunsch, 1990). Although Aki (1993) emphasized its potential in the overview of an early encyclopedic book on seismic tomography (Iyer and Hirahara, 1993), it has been used only in a few studies in earthquake seismology. Zeng (1991) used this scheme to obtain a spatial distribution of site effects in his coda analysis under the name of a "recursive stochastic inversion method". Nishigami (2000) inverted the spatial distribution of scattering strength along and around the San Andreas fault in central California with temporal and spatial variations of coda envelopes observed by a seismic network in this area, using the same scheme. These studies emphasized the advantage of this scheme in terms of (a) its computational efficiency for a large matrix system and (b) the simultaneous computation of its resolution and error covariance matrices.

We shall briefly review the scheme here, in order to observe its merits in the application to real-time seismic tomography. Let us first consider two independent measurements of model, $m_{1}$ and $m_{2}$, with variances of $\sigma_{1}^{2}$ and $\sigma_{2}^{2}$ for a uncertain scalar, $m$. A weighted average of $m$, denoted by $\hat{m}$, is expressed by

$$
\hat{m}=\frac{\frac{m_{1}}{\sigma_{1}^{2}}+\frac{m_{2}}{\sigma_{2}^{2}}}{\frac{1}{\sigma_{1}^{2}}+\frac{1}{\sigma_{2}^{2}}}
$$

with its variance given by

$$
\hat{\sigma}^{2}=\frac{1}{\frac{1}{\sigma_{1}^{2}}+\frac{1}{\sigma_{2}^{2}}}
$$

Next, we generalize the above scalar representation to the vectorial one in a straightforward manner. Suppose that we have two measurements, $\mathbf{m}_{1}$ and $\mathbf{m}_{2}$, for a model parameter vector $\mathbf{m}$ with their model or a priori covariance matrices, $\mathbf{S}_{1}$ and $\mathbf{S}_{2}$, respectively. The estimated value of $\mathbf{m}$ is expressed by

$$
\hat{\mathbf{m}}=\left(\mathbf{S}_{1}^{-1}+\mathbf{S}_{2}^{-1}\right)^{-1}\left(\mathbf{S}_{1}^{-1} \mathbf{m}_{1}+\mathbf{S}_{2}^{-1} \mathbf{m}_{2}\right)
$$

with the corresponding covariance matrix given by

$$
\hat{\mathbf{S}}=\left(\mathbf{S}_{1}^{-1}+\mathbf{S}_{2}^{-1}\right)^{-1} .
$$

Now let us apply the above result to Eq. (3) in seismic tomography. We introduce the covariance matrices, $\mathbf{S}_{\varepsilon}$ and $\mathbf{S}_{p}$, of the data and model parameters, $\delta \mathbf{d}$ and $\delta \mathbf{m}_{p}$, respectively, and substitute them into Eqs. (4) and (5). Equation (3) can be then solved in terms of stochastic inverse (e.g., Aki and Richards, 1980) in the following form:

$$
\delta \hat{\mathbf{m}}=\left(\mathbf{S}_{p}^{-1}+\mathbf{G}^{T} \mathbf{S}_{\varepsilon}^{-1} \mathbf{G}\right)^{-1}\left(\mathbf{S}_{p}^{-1} \delta \mathbf{m}_{p}+\mathbf{G}^{T} \mathbf{S}_{\varepsilon}^{-1} \delta \mathbf{d}\right)
$$

with the covariance

$$
\hat{\mathbf{S}}=\left(\mathbf{S}_{p}^{-1}+\mathbf{G}^{T} \mathbf{S}_{\varepsilon}^{-1} \mathbf{G}\right)^{-1}
$$

Equations (8) and (9) can be rewritten as follows:

$$
\delta \hat{\mathbf{m}}=\delta \mathbf{m}_{p}+\mathbf{S}_{p} \mathbf{G}^{T}\left(\mathbf{G S}_{p} \mathbf{G}^{T}+\mathbf{S}_{\varepsilon}\right)^{-1}\left(\delta \mathbf{d}-\mathbf{G} \delta \mathbf{m}_{p}\right)
$$

$$
\hat{\mathbf{S}}=\mathbf{S}_{p}-\mathbf{S}_{p} \mathbf{G}^{T}\left(\mathbf{G S}_{p} \mathbf{G}^{T}+\mathbf{S}_{\varepsilon}\right)^{-1} \mathbf{G} \mathbf{S}_{p}
$$

The direct use of the above equations requires the computation of an inverse matrix, $\left(\mathbf{G S}_{p} \mathbf{G}^{T}+\mathbf{S}_{\varepsilon}\right)^{-1}$, which is of the $N \times N$-dimension. Note that most of recent travel-time inversions adopt an iterative solution of the matrix system of Eq. (3) without the use of any inverse matrices (e.g., Nolet, 1987; Iyer and Hirahara, 1993).

The recursive inverse scheme summarized by Rodgers (1976), called sequential estimation there, modifies it into a series of simple formulations for each data set (i.e., $\delta d_{i}$ or each element of the data vector $\delta \mathbf{d}$ ). For simplicity, we assume non-diagonal components of the data covariance matrix to be zero, that is, $\mathbf{S}_{p}$ consists only of $\sigma_{i}^{2}$ as the $i$-th diagonal component. Since $\sigma_{i}^{2}$ corresponds to the variance of the $i$-th measurement $\delta T_{i}$, this simplification means no correlations among errors or noise in data and does not become a strong restriction in seismic tomography in practice. Denoting $\mathbf{g}_{i}$ to be the $i$-th row vector of the matrix G, Eqs. (10) and (11) are expressed by the following series $(i=1,2, \ldots, N)$ of equations in a recursive form, or equivalent to equation (69) in Rodgers (1976):

$$
\delta \hat{\mathbf{m}}_{i}=\delta \hat{\mathbf{m}}_{i-1}+\left(\frac{\mathbf{S}_{i-1} \mathbf{g}_{i}^{T}}{\mathbf{g}_{i} \mathbf{S}_{i-1} \mathbf{g}_{i}^{T}+\sigma_{i}^{2}}\right)\left(\delta d_{i}-\mathbf{g}_{i} \delta \hat{\mathbf{m}}_{i-1}\right)
$$

with the model covariance

$$
\mathbf{S}_{i}=\mathbf{S}_{i-1}-\left(\frac{\mathbf{S}_{i-1} \mathbf{g}_{i}^{T} \mathbf{g}_{i} \mathbf{S}_{i-1}}{\mathbf{g}_{i} \mathbf{S}_{i-1} \mathbf{g}_{i}^{T}+\sigma_{i}^{2}}\right)
$$

The initial value of $\delta \hat{\mathbf{m}}, \delta \hat{\mathbf{m}}_{0}$, is zero while $\mathbf{S}_{0}$ may be selected with any a priori information of model parameters in the same sense as stochastic inversion. The choice of $\mathbf{S}_{0}$ corresponds to the size of the damping factor in the case of an inversion of least-squares (e.g., Aki and Richards, 1980). Their final values corresponds to the estimated values of model parameters and model covariance matrix:

$$
\delta \hat{\mathbf{m}}=\delta \hat{\mathbf{m}_{N}}, \quad \hat{\mathbf{S}}=\mathbf{S}_{N}
$$

As pointed out by Zeng (1991) and Nishigami (2000), the above formulations requires only the division of one scalar in each recursive step, as easily confirmed by Eqs. (12) and (13). This saves the computational amount significantly because the numbers of both data and model parameters, $N$ and $M$, are very large in recent travel-time inversions. It also naturally introduces the corresponding model covariance matrix, that is, errors in the estimation of model parameters.

The above formulations have an important character suitable for the purpose of real-time seismic tomography. In Eqs. (12) and (13), we process $\delta d_{i}$, that is, each piece of our data, using the result of the model parameters inverted by the previous stage of the inversion with the data from $\delta d_{1}$ to $\delta d_{i-1}$. Suppose that we have already obtained the model parameters with the data set of the total number $N$. Then, if we obtain a new data set, named $\delta d_{N+1}$, we only need to add one simple computation of Eqs. (12) and (13) for $i=N+1$, with a vector $\mathbf{g}_{N+1}$ that corresponds to the additional ray path for the $(N+1)$-th observation. As shown in Eq. (12), we obtain a new result $\delta \hat{\mathbf{m}}_{N+1}$ in the form of the 

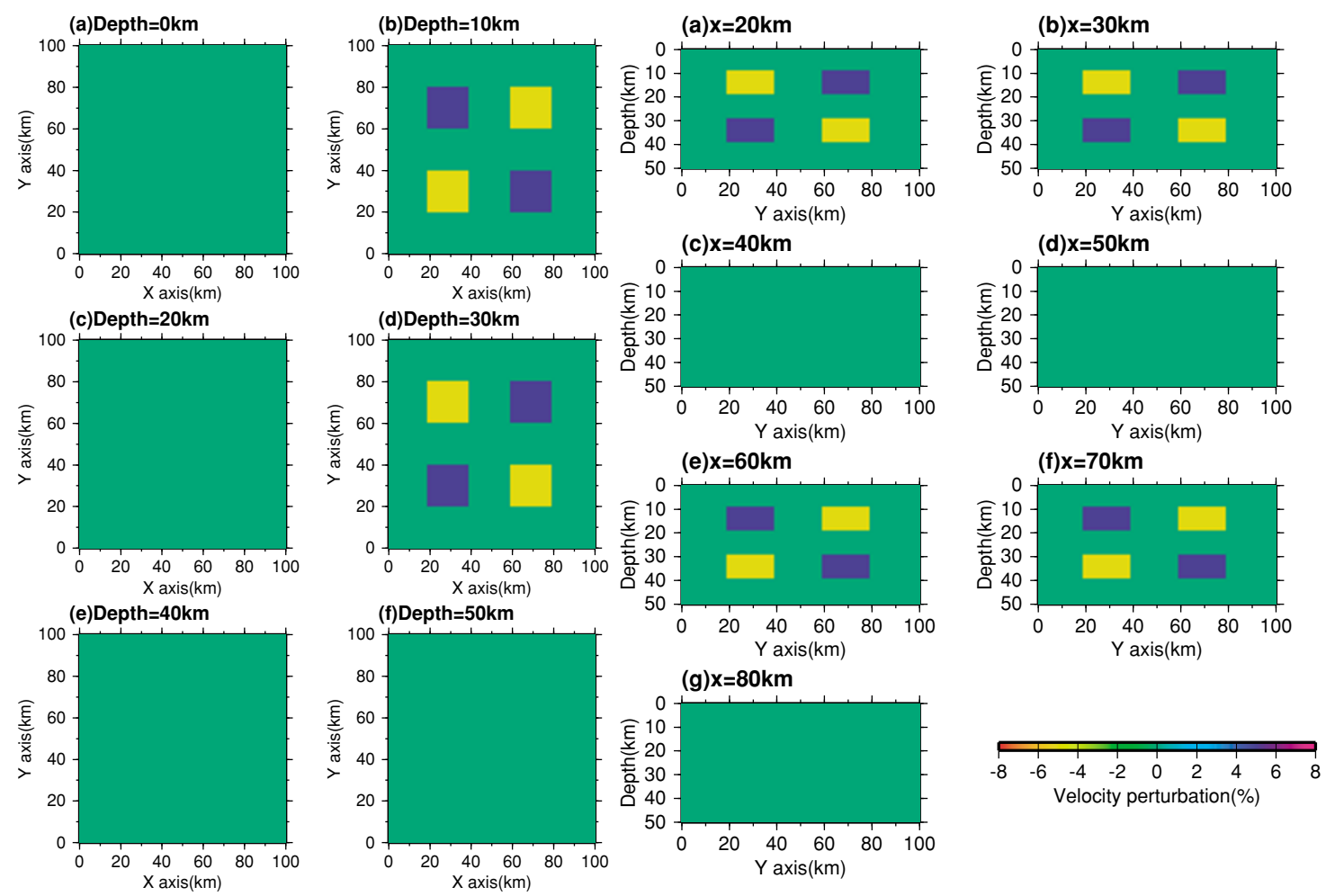

Fig. 1. Depth slices and cross sections along the $x$ axis of the assigned velocity model for a numerical test.
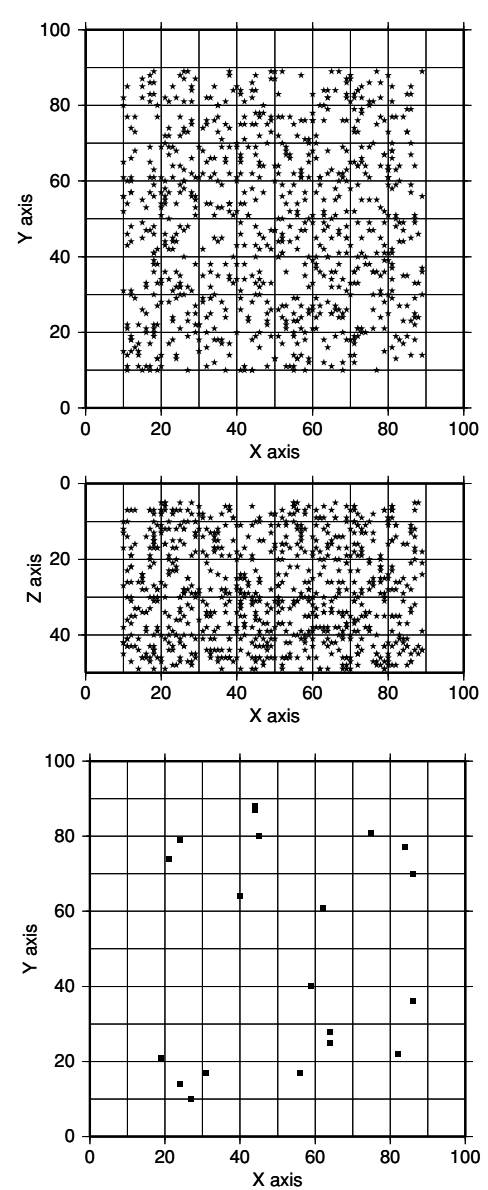

Stations

Fig. 2. Source and station distributions used for a numerical test. revision of the previous model $\delta \hat{\mathbf{m}}_{N}$. This feature is suitable to real-time seismic tomography in practice, as explained in Introduction.

Besides the small amount of additional computations when a new piece of data becomes available, this scheme has another advantage over the conventional ones: the reference velocity model for computing a ray path at each recursive step can be updated in a straightforward manner, while we must use the same initial velocity model for all the ray paths in conventional inverse schemes. In the conventional ones, we may revise the velocity model after the first result is inverted, and then obtain the revised ray paths and travel times for such a new model, repeating this process until the result converges. This approach, however, requires a lot of computations, and not practical in the points of view of real-time seismic tomography.

\section{Test with Synthetic Data}

In order to investigate the accuracy, stability and efficiency of our proposed inverse scheme in travel-time tomography, we shall conduct a test with the data synthesized for a given three-dimensional velocity model. It is important to observe not only how accurately our inversion scheme can retrieve the given velocity model but also whether its result is different from the one obtained by a conventional inverse scheme or not.

The background velocity structure of the model varies with depth as $v(z)=6.0+0.04 \times z$, where $v$ and $z$ represent velocity in $\mathrm{km} / \mathrm{s}$ and depth in $\mathrm{km}$, respectively, in a 100 $\mathrm{km} \times 100 \mathrm{~km} \times 50 \mathrm{~km}$ model region. We added $\pm 5 \%$ positive or negative velocity perturbations as the total of eight blocks of $20 \mathrm{~km} \times 20 \mathrm{~km} \times 10 \mathrm{~km}$ size, as shown in Fig. 1 . 

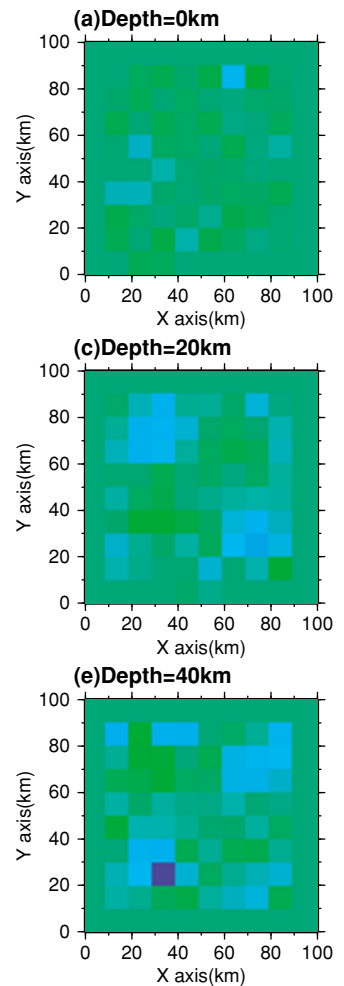

(b) Depth $=10 \mathrm{~km}$
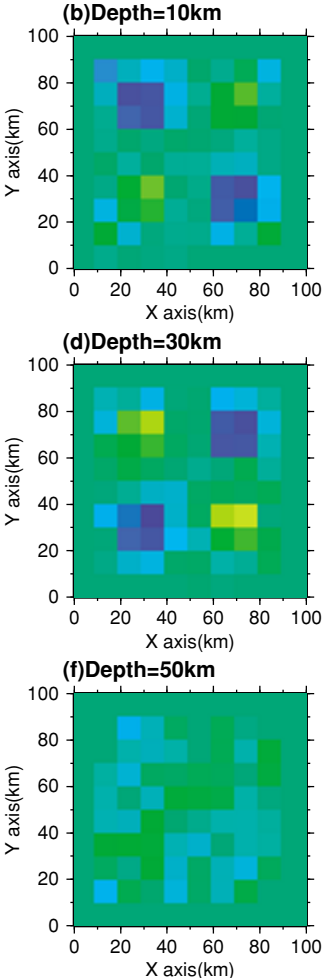

(a) $x=20 \mathrm{~km}$

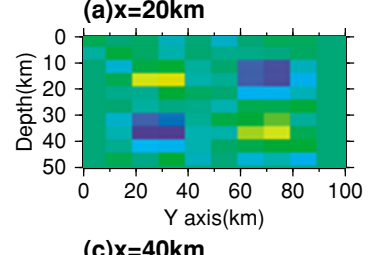

(c) $x=40 \mathrm{~km}$

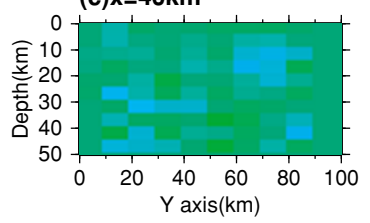

(e) $x=60 \mathrm{~km}$

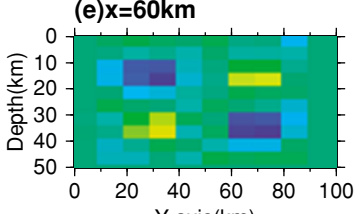

(g) $x=80 \mathrm{~km}$

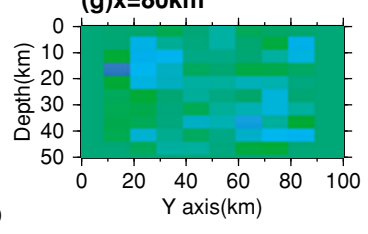

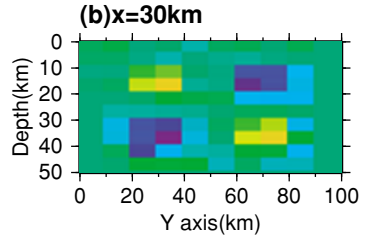

(d) $x=50 \mathrm{~km}$
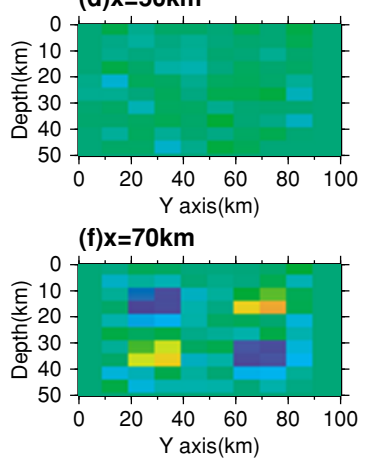

$\begin{array}{lllllllll}-8 & -6 & -4 & -2 & 0 & 2 & 4 & 6 & 8\end{array}$

Velocity perturbation(\%)

Fig. 3. Depth slices and cross sections along the $x$ axis inverted by the conventional inverse scheme.

As a rather ideal situation, sources were distributed in a relatively uniform manner over the model space while stations are assigned sparsely, as shown in Fig. 2. The total number of the synthetic travel-time data is 16,000 . Synthetic travel-times from source to station were calculated by the Huygens' method, as explained in the previous section. We divided the model space into cubic blocks of $1 \mathrm{~km} \times 1 \mathrm{~km}$ $\times 1 \mathrm{~km}$ size for ray tracings.

Velocity perturbations were inverted at grid points at 10 $\mathrm{km} \times 10 \mathrm{~km} \times 5 \mathrm{~km}$ intervals in the model space. We conducted the following two types of inversion: (a) conventional iterative inverse scheme called the LSQR algorithm of Paige and Saunders (1982) that was adopted in Zhao et al. (1991) and Katsumata et al. (2003), and (b) recursive inverse scheme explained in the previous section. Note that all the ray tracings were conducted for the same initial velocity model in (a) with several iterations for each updated model, while the reference velocity model for ray tracing was revised at each recursive step, or each $i$-th data set in Eq. (12), in (b).

Figure 3 shows the final result of three-dimensional velocity perturbations obtained by the conventional inverse scheme while Fig. 4 by the recursive inverse scheme. Remember that there is one important a priori parameter in each scheme. In other words, each final result may be strongly affected by a rather subjective choice of such parameters. In the conventional iterative inversion, we must judge the convergence of its result to terminate its iteration process. In contrast, we need to select the initial model covariance matrix $\mathbf{S}_{0}$ in Eqs. (12) and (13) in the recursive inverse scheme. The size of its diagonal elements is closely related to a damping factor in the sense of least-squares, as mentioned in the previous section. In the former case, we terminated the iteration process so that the velocity distribution such as Fig. 3 does not alter significantly even if we repeat more iterations. In the latter case, we obtained results for several different sizes of $\mathbf{S}_{0}$, judging the optimal one as the smallest of all that do not show obvious instability in result, similar to the standard stochastic inversion (e.g., Aki and Richards, 1980).

Both schemes successfully retrieve the assigned velocity perturbations in Fig. 1, showing that our recursive inverse scheme can retrieve a reliable velocity structure similar to the one obtained by the conventional inverse scheme. In addition, the recursive inverse scheme gives a somehow better final result than the conventional one, particularly in a deep part, for example, at depth of $40 \mathrm{~km}$ (Figs. 3 and 4). This difference is mainly originated not due to the theoretical superiority in scheme but the introduction of the updated reference velocity model at each recursive step. Many rays in the present problem, particularly for sources shallower than $30 \mathrm{~km}$, have their turning points near the bottom of the model space (Fig. 2). The accuracy of the reference model strongly affects ray paths and travel times for rays propagating laterally in a vertically varying medium, that is, where their turning points are located. That is one major reason why a vertical resolution is generally worse than the horizontal in seismic tomography with local events (e.g., Aki and Lee, 1976). Since the recursive inverse scheme revised the velocity model more frequently than the conventional (i.e., each recursive step versus each iteration with all the data combined), more precise ray paths and travel times were calculated, particularly for rays with turning points. 

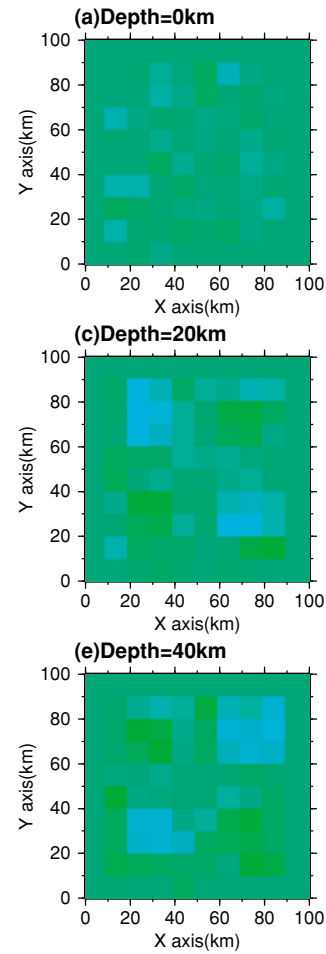
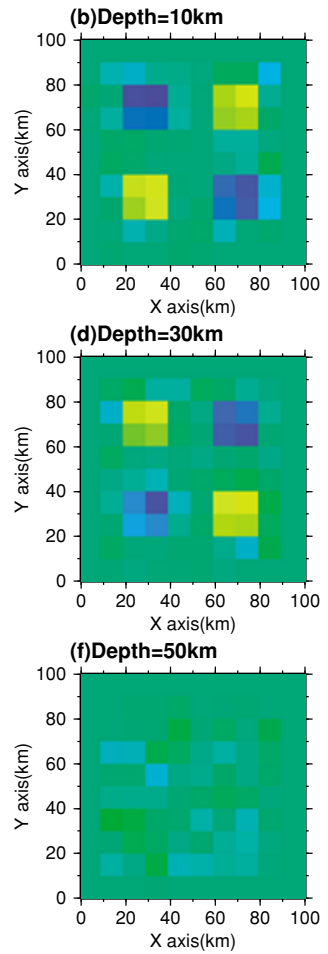
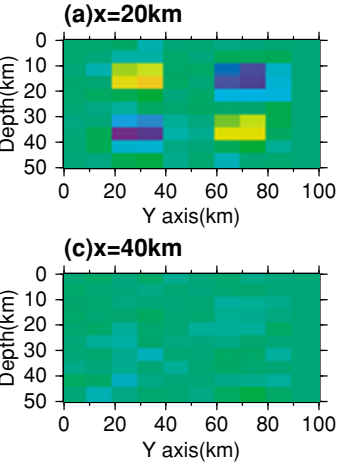

(e) $x=60 \mathrm{~km}$

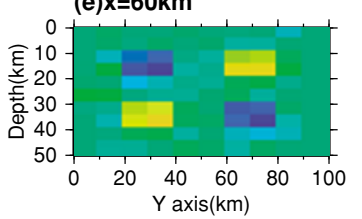

(g) $x=80 \mathrm{~km}$

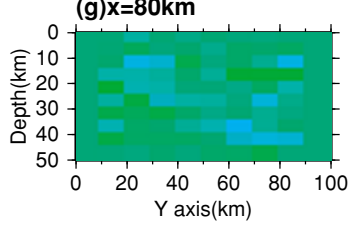

(b) $x=30 \mathrm{~km}$

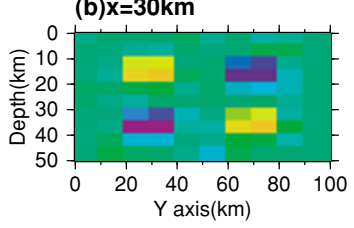

(d) $x=50 \mathrm{~km}$

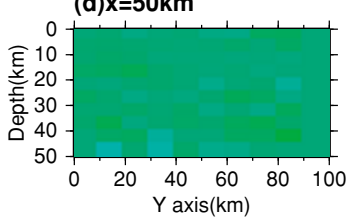

(f) $x=70 \mathrm{~km}$

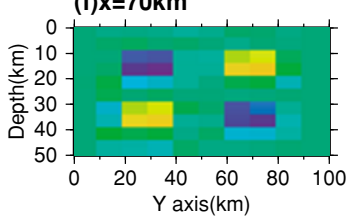

$\begin{array}{lllllllll}-8 & -6 & -4 & -2 & 0 & 2 & 4 & 6 & 8\end{array}$

Velocity perturbation(\%)

Fig. 4. Depth slices and cross sections along the $x$ axis inverted by the recursive inverse scheme.

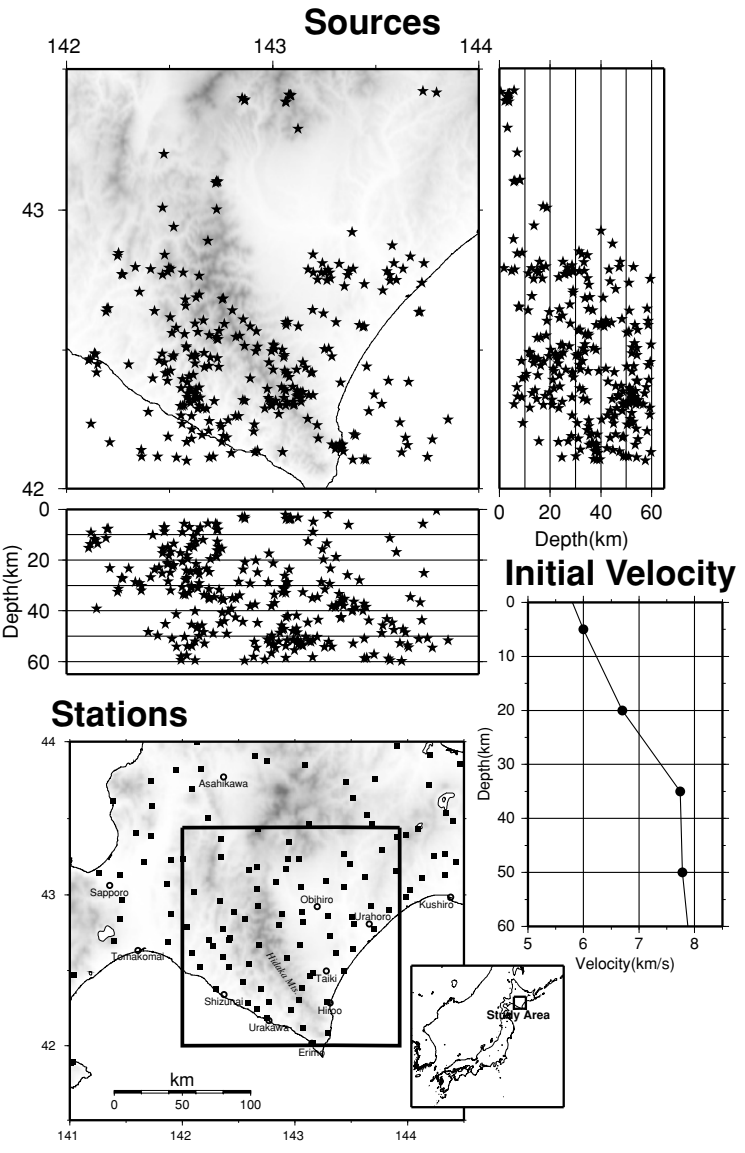

Fig. 5. Distributions of earthquakes (above) and station locations (bottom). The square of thick lines in the bottom represents the model space. Squares indicate the stations, while circles for major cities and towns. The initial velocity model for inversion is shown in the right, with dots denoting the grid points used in Katsumata et al. (2003).

\section{Application to the Velocity Structure in the Hi- daka Region}

We applied the proposed recursive inverse scheme to the $P$-wave velocity structure in the Hidaka, Hokkaido, Japan, region where the Kuril island arc and the Northeastern Japan arc collide each other. Seismicity there is high (Moriya et al., 1997), mainly related to the de-lamination of the Kuril island arc under the mountains (e.g., Murai et al., 2003; Taira and Yomogida, 2004). Takanami (1982) and Miyamachi and Moriya $(1984,1987)$ estimated its threedimensional velocity structure by a tomographic approach. Moriya et al. (1998), Iwasaki et al. (1998), Tsumura et al. (1999), and Iwasaki et al. (2003), on the other hand, obtained its fine crustal structure with dense seismic refraction and/or reflection profiles.

From July 1997 to July 2001, a research group from eleven universities in Japan operated a dense temporary seismic network in and around this region (Katsumata et al., 2002). Using the seismograms recorded by this network, three-dimensional $P$ - and $S$-wave velocity structures were obtained in this tectonically interesting area with one of the highest resolutions for tomographic results in Japan (Katsumata et al., 2003). In this study, we used a part of this large travel-time data set. We shall compare our result with Katsumata et al. (2003) obtained by the conventional iterative tomographic scheme (Paige and Saunders, 1982; Zhao et al., 1992) with the entire data set, so that we check the accuracy and efficiency of our proposed inversion approach with actual travel-time data.

\subsection{Data}

Distributions of seismic stations and earthquakes are shown in the bottom of Fig. 5, as a part of the data set used by Katsumata et al. (2003), focusing here only on the region 

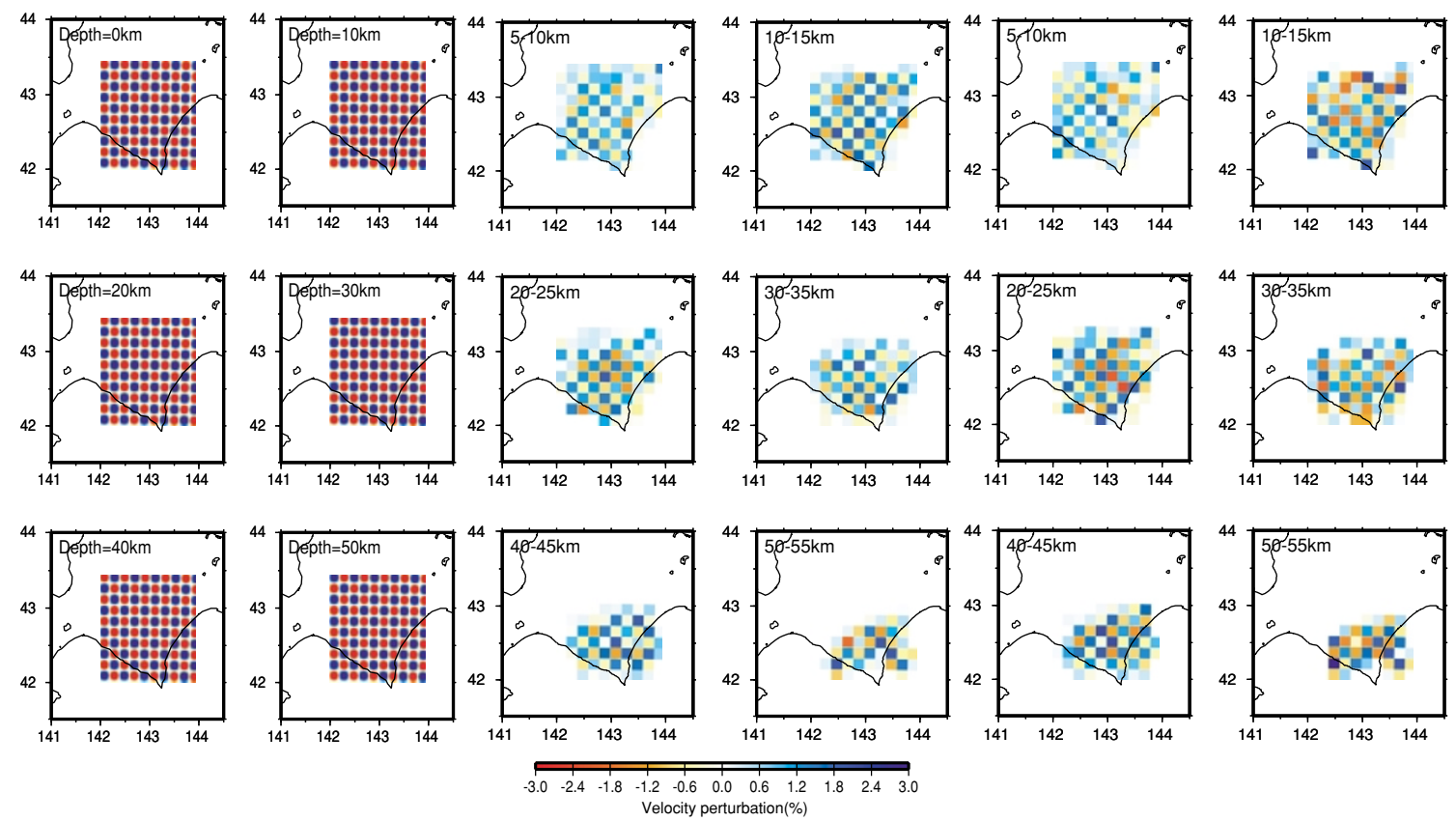

Fig. 6. Assigned checkerboard pattern (left), the result by the conventional inverse scheme (middle) and that by the recursive inverse scheme (right) in depth slices.

designated by the square in Fig. 5, from 0 to $60 \mathrm{~km}$ deep, in comparison with the original model space of Katsumata et al. (2003). These earthquake locations were routinely determined, using the data set recorded by the Hokkaido University Seismic Network (Katsumata et al., 2002). Similar to Katsumata et al. (2003), we did not apply any station correction terms to the observed travel-time data because they showed that the resulting velocity structure was affected little by their introduction. The data in this study consists of 7,366 $P$-wave travel times for 282 earthquakes.

The one-dimensional velocity structure same as that of Katsumata et al. (2003) was used as the initial model, except for the omission of a surface layer shallower than $5 \mathrm{~km}$, as shown in the right of Fig. 5. We corrected this velocity model by the earth-flattening transformation (e.g., chapter 4 of Shearer, 1999) in our inversion. For ray tracings, we divided the model space of $160 \mathrm{~km} \times 160 \mathrm{~km} \times 60 \mathrm{~km}$ size (Fig. 5) into grids at $1 \mathrm{~km} \times 1 \mathrm{~km} \times 1 \mathrm{~km}$ intervals. We used rectangular blocks of $16 \mathrm{~km} \times 16 \mathrm{~km} \times 5 \mathrm{~km}$ size for the model parameters to be inverted. The standard deviation of travel-time residuals of the present data set is $0.2450 \mathrm{sec}$ for the initial velocity model.

\subsection{Checkerboard Test}

In order to confirm the accuracy and resolution of the present data set, we performed a checkerboard test at first. We assumed the maximum of $\pm 10 \%$ velocity perturbations at grid points with an even interval of $16 \mathrm{~km}$ and $5 \mathrm{~km}$ in the horizontal and vertical directions, respectively. The velocity at each grid point for ray tracings was assigned as a linear interpolation of the above velocity perturbation. In the left of Fig. 6, depth slices of the assigned velocity model are shown. Note that we assumed the maximum of $\pm 10 \%$ perturbations while $\pm 3 \%$ perturbations in the color scale of this figure, because the given slices do not include any points of maximum perturbations. We created synthetic travel-time data with this velocity model for the same source-receiver pairs as the observed travel-time data. Other parameters such as the choice of the initial model covariance matrix $\mathbf{S}_{0}$ were same as those used for the numerical test in the previous section.

The final three-dimensional velocity models obtained by the conventional and recursive inverse schemes are shown in the middle and right of Fig. 6, respectively, in the form of depth slices. Both schemes can retrieve the assigned checkerboard pattern quite well, although the absolute values of velocity perturbations are suppressed in either a case, due to the effect of damping. We can nevertheless notice slight difference in their obtained patterns, as follows. At depth of $10 \sim 15 \mathrm{~km}$, the recursive tomography can retrieve both positive and negative perturbations, while negative perturbations (i.e., orange boxes) around the northeastern corner of the model space are not retrieved sufficiently in the conventional one. As seen in Fig. 5, the number of ray paths are limited in this area, due to weak seismicity there. Similar to the result of the previous numerical test, the use of the updated velocity model at each recursive step provides a slightly better result than the conventional scheme in an area of the model space where the coverage of ray paths is not sufficient. The conventional inverse scheme would provide the same good result if we re-traced all the rays with the updated velocity model and repeat this procedure many times.

\subsection{Effect of the order of data}

Since the entire data set is considered together in any conventional inverse schemes, the order of data (i.e., the subscript $i$ for the data vector $\delta d_{i}$ in Eq. (3)) does not affect the final result. Even with the use of an iterative scheme, solutions should converge into a single final result after many times of iterations. In contrast, the present recursive inverse scheme solves the problem gradually as one piece of data 
data A
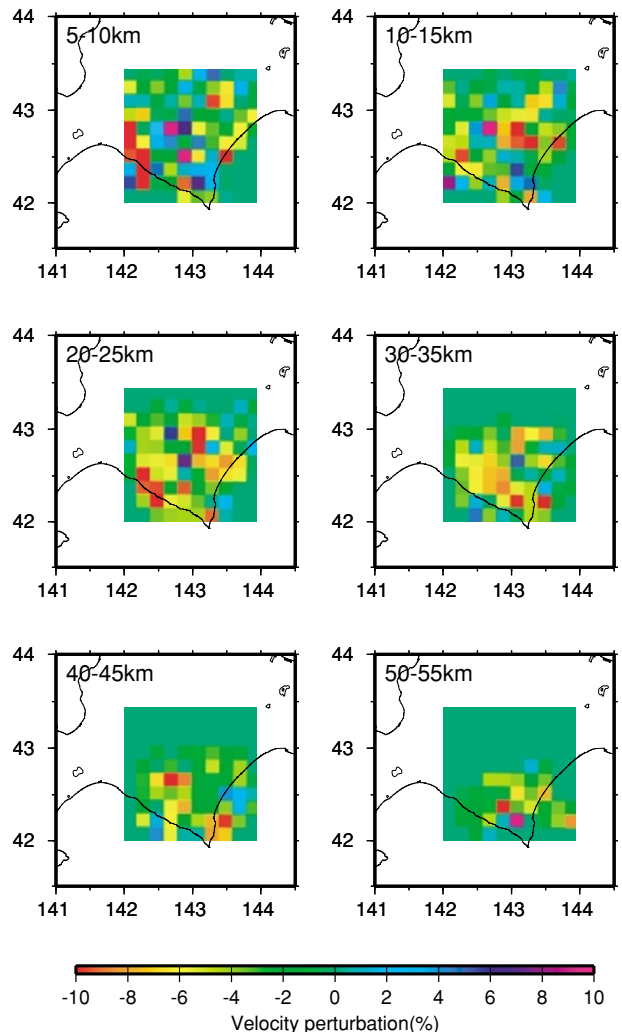

data B
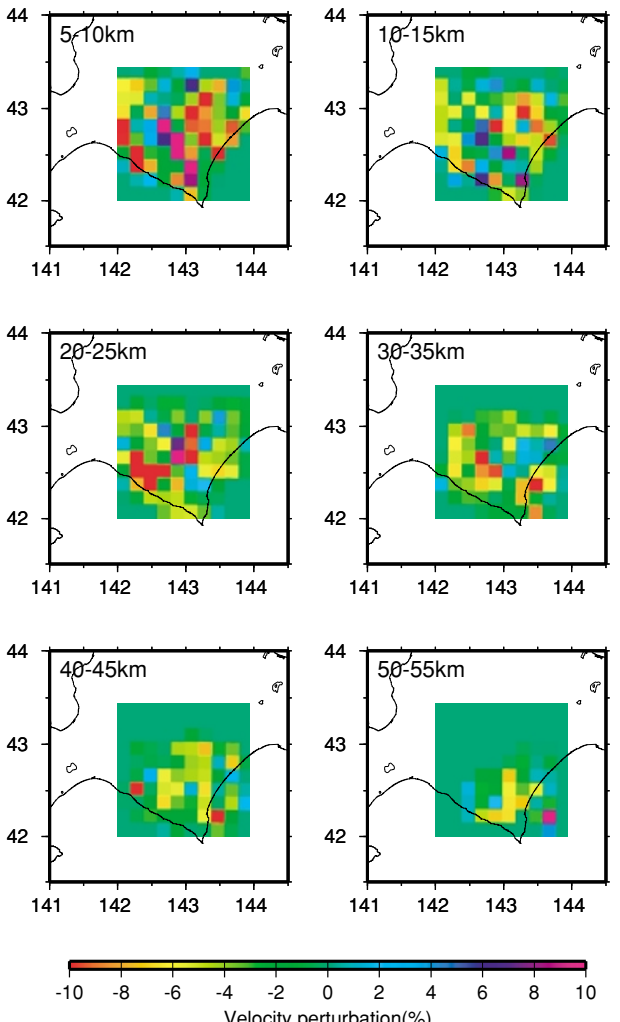

Fig. 7. Depth slices of the velocity structures obtained by the recursive inverse scheme, using only a half of the entire data, called data $A$ (left) and data $B$ (right).
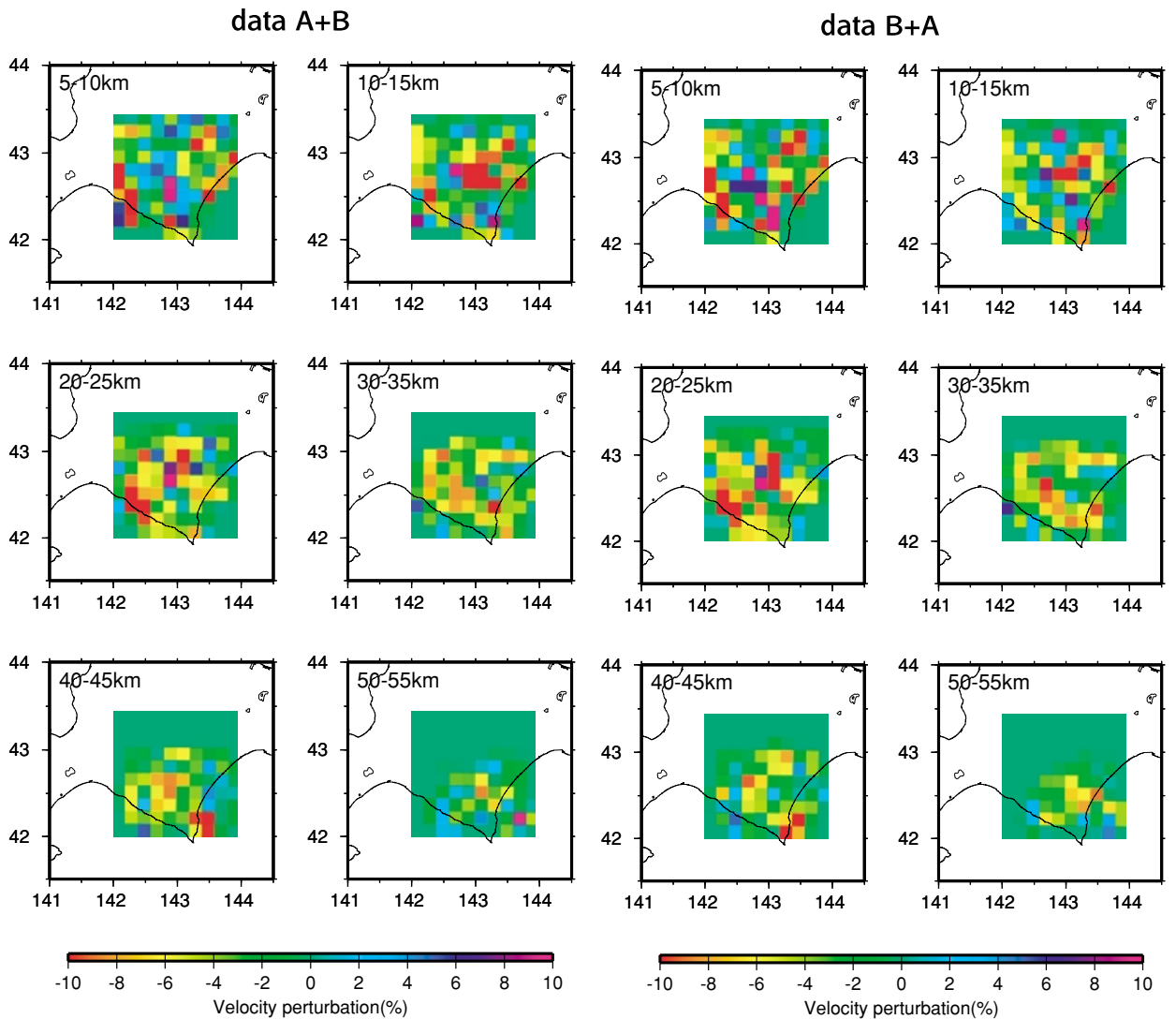

Fig. 8. Depth slices of the velocity structures obtained by the recursive inverse scheme, using data $A$ first and data $B$ later (left) and using data $B$ first and data $A$ later (right). 
is added, as shown in Eqs. (12) and (13). That is why we propose this scheme to be suitable for the detection of temporal variations in velocity in real-time seismic tomography in future. In other words, the order of the data is a key factor in this scheme.

In this subsection, we shall test the accuracy and reliability of the proposed recursive inverse scheme for different orders of the same data set. If the scheme works well, the final result should be the same even though intermediate results with a part of the data set are different.

We divided the total of 282 events into two halves (i.e., 141 events for each sub data set), called data $A$ and data $B$, where the events of data $A$ took place first. We then obtained a three-dimensional distribution of velocity perturbations in the Hidaka area, using either data $A$ or $B$. Figure 7 shows each result in the form of depth slices. The result at depths with relatively sufficient ray paths such as depths of 10 and $20 \mathrm{~km}$ is similar to each other while the result at depths of more than $30 \mathrm{~km}$ is quite different. There are little ray paths in a deep part, and the regions sampled sufficiently are different between the two sub data sets. One may interpret the difference between them as temporal variation of the velocity structure in this region. We do not deny the possibility of temporal variation, but the difference in the coverage of ray paths is likely to be the main reason for the difference in Fig. 7.

Figure 8 shows the final results with different orders of data in the recursive inverse scheme. The left figure shows the result when we first inverted data $A$ (i.e., the left of Fig. 7) and subsequently did data $B$. On the other hand, the right shows the result in the case of data $B$ first (i.e., the right of Fig. 7), followed by data $A$. Although there are areas of slightly different velocity perturbations, the agreement between them is sufficient if we consider the general resolution of seismic tomography these days. The results in a deep part (e.g., $40 \mathrm{~km}$ deep) are now similar to each other although each half of the entire data set gives clearly different results, as shown in Fig. 7.

Remember that we update the reference velocity model for the ray tracing at each recursive step. The velocity model is updated, following the result up to the previous recursive step. The evolution of reference models naturally depends on the order of data although its effect should not be very large. In other words, the ray path to be inverted at a given piece of data (i.e., one observation of travel time) slightly depends on the order of data. This factor should be a part of reasons for the difference between the two results of Fig. 8, so that we can claim that actual numerical errors or instabilities in the proposed recursive inverse scheme are less than those explicitly appearing in Fig. 8.

In summary, although the recursive inverse scheme of Eqs. (12) and (13) apparently depends on the order of data strongly, the present test proves that the final result is invariant, that is, accurate and reliable. This test also shows the potentiality in the detection of temporal variations in velocity, whether difference of the two figures of Fig. 7 is reliable or not, after taking the quality of data into consideration.

\subsection{Results}

Finally, let us investigate the three-dimensional $P$-wave velocity structure in the Hidaka region, using the recursive inverse scheme in comparison with the conventional one. The velocity structure imaged by the conventional one is shown in Fig. 9, while Fig. 10 by the recursive one. In the recursive one, we sorted the order of data different from the previous two cases of Fig. 8. While the variance reduction of travel-time data is $48.8 \%$ for the conventional scheme, $61.6 \%$ for the recursive one, slightly larger, due to the use of the updated reference velocity model at each recursive step, as explained above. The total computational times of both methods are of the same order, as far as we obtain the results of similar spatial resolution, as shown in these figures. As predicted, both results agree well each other, confirming the accuracy and stability of our proposed recursive inverse scheme even with actual data.

At a depth of $5 \mathrm{~km}$, there are zones of high velocity in the center of the region, surrounded by zones of low velocity. This high-velocity zone is located nearly parallel to the Hidaka mountains. The west side of the low-velocity zones seems to be subducting from west to east, as seen in the cross section at $42.2 \mathrm{~N}$ of Figs. 9 and 10. Figure 10, however, shows this subducting image more clearly. We can also identify another low-velocity body in the east of the Hidaka Mountains at a depth of $10 \mathrm{~km}$ in the recursive scheme (Fig. 10) but not the conventional (Fig. 9), which will be discussed in the next subsection.

\subsection{Comparison with previous results}

Figure 11 shows the three-dimensional velocity model obtained by Katsumata et al. (2003). Since we used only a part of their travel time data set, the agreement between our result (Fig. 10) and theirs (Fig. 11) with the conventional iterative inverse scheme of Zhao et al. (1992) reconfirms the validity and accuracy of the recursive inverse scheme even with actual travel-time data with observational noise. For example, a low-velocity zone exists in both results, extending from Urakawa to Erimo at a depth of about 20 $\mathrm{km}$.

Nevertheless, we can identify slight discrepancies between them, and we need to investigate their origins. While a high-velocity zone in Urakawa-Oki (i.e., along the southwestern off-shore of south-central Hokkaido) about $20 \mathrm{~km}$ deep is recognized in Katsumata et al. (2003), this study could not retrieve such a feature of high velocity. We used few travel-time data passing through this area (see Fig. 5), in order to limit our model space to be sampled sufficiently by ray paths. The lack of our detectability of the above feature is not due to the incompleteness of the recursive inverse scheme but originated from the quality of data.

In contrast, our result at a depth of $10 \mathrm{~km}$ clearly shows a strong low-velocity area in the center of the model space, as mentioned in the end of the previous subsection. Although the grid interval of Katsumata et al. (2003) is slightly larger than ours and they did not show a result around $10 \mathrm{~km}$ deep, there does not seem to exist such a low-velocity area in the corresponding location even by the results at adjacent depths (i.e., 5 and $20 \mathrm{~km}$ deep) of Fig. 11. While we performed ray tracings for a revised reference velocity structure at each recursive step, the result of Katsumata et al. (2002) was derived after only several iterations through the whole data set, using the revised reference velocity model at each iteration. The number of these iterations cannot be 

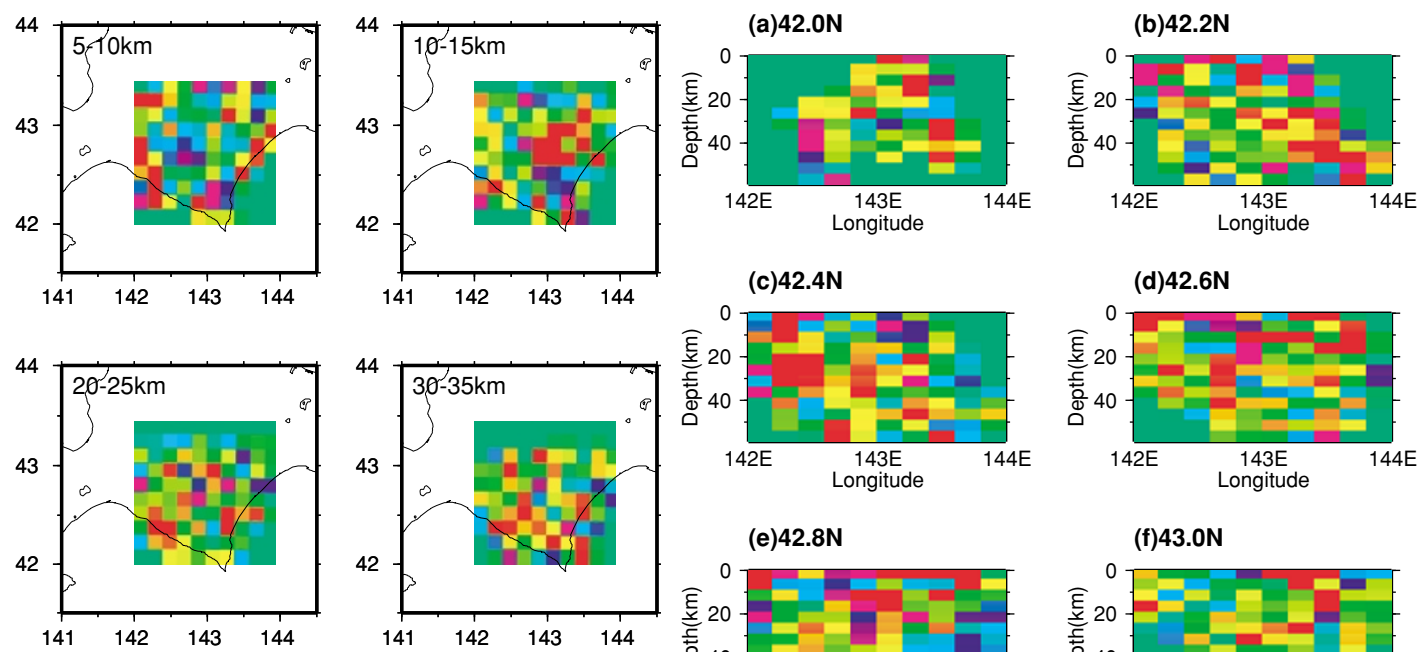

(d) $42.6 \mathrm{~N}$

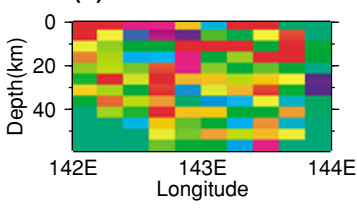

(e) $42.8 \mathrm{~N}$

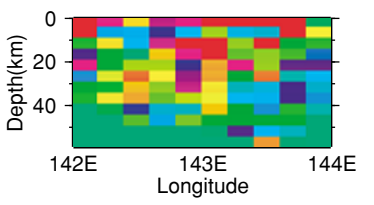

(f) $43.0 \mathrm{~N}$
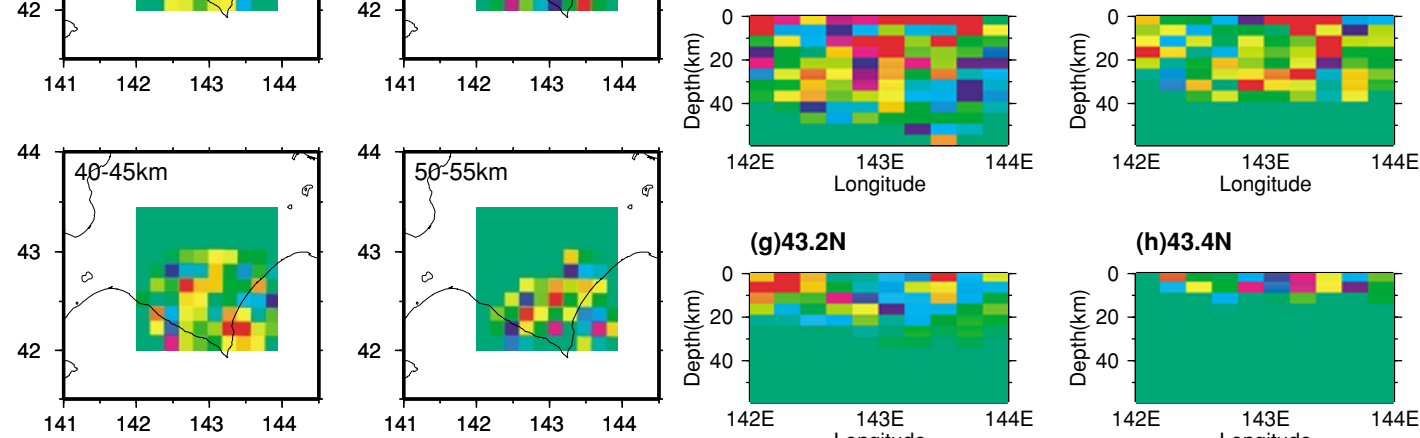

(g) $43.2 \mathrm{~N}$

(h) $43.4 \mathrm{~N}$
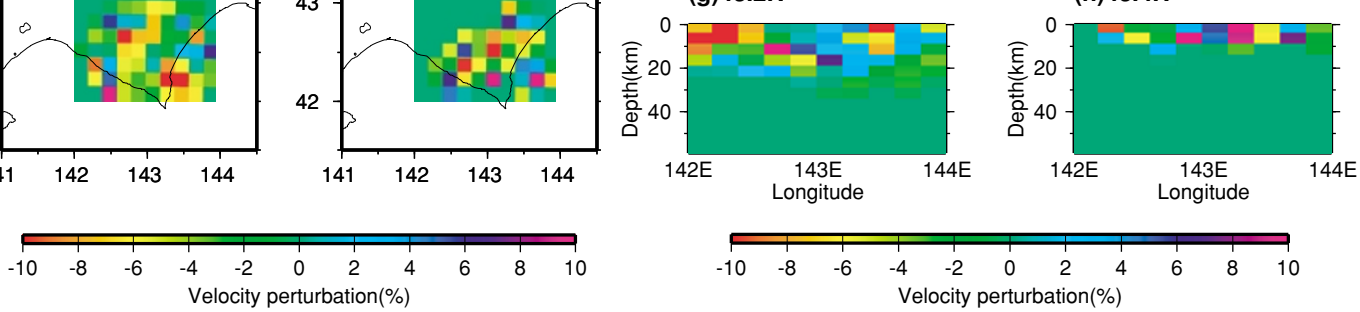

Fig. 9. Depth slices and east-west cross sections of the velocity structure obtained by the conventional inverse scheme.
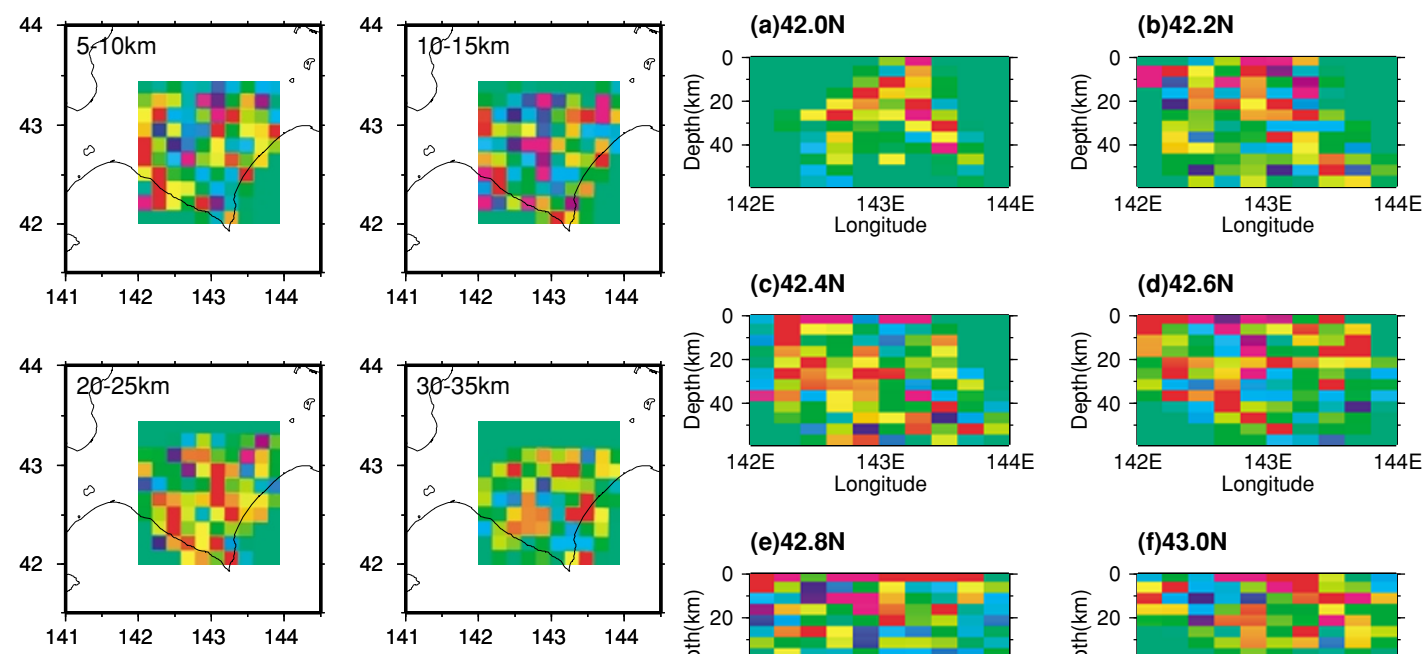

(e) $42.8 \mathrm{~N}$

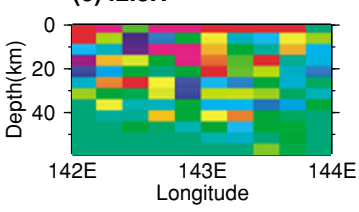

(g) $43.2 \mathrm{~N}$

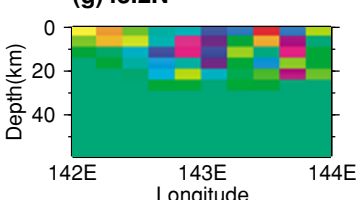

(f) $43.0 \mathrm{~N}$

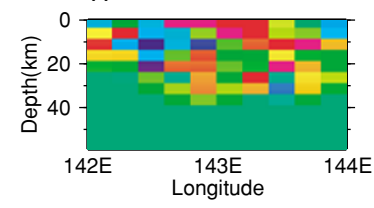

(h) $43.4 \mathrm{~N}$

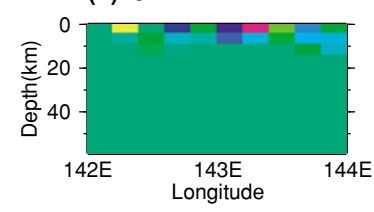

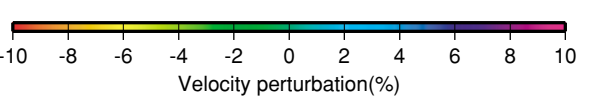

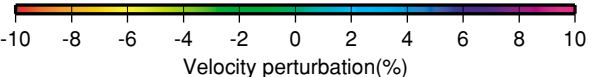

Fig. 10. Depth slices and east-west cross sections of the velocity structure obtained by the recursive inverse scheme. 
increased easily because that would require the total amount of calculations multiplied by the iteration number. This means that our recursive inverse scheme can easily obtain the above feature of high resolution by the natural introduction of updated velocity models without increasing its computational time.

In 1999 and 2000, extensive refraction/wide-angle reflection surveys were conducted in this region. Iwasaki et al. (2003) obtained a very detailed $P$-wave velocity profile in the crust along an east-west line across the Hidaka Mountains. At depth of about $10 \mathrm{~km}$, they found a region of low velocity $(5.5-6.5 \mathrm{~km} / \mathrm{s})$ with strong seismic reflection in the east of the axis of the Mountains. They interpreted it as the lower crust of the Kuril arc thrusting over the Northeastern Japan arc, due to their collision there. The body of low velocity at the same depth retrieved by the recursive inverse scheme (Fig. 10) seems to correspond to the uppermost layer of the subducting arc, or the reflector above the collision zone, obtained by Iwasaki et al. (2003).

\section{Conclusions}

We proposed a new inversion scheme for travel-time seismic tomography, combining ray tracing by the Huygens' method of Saito (2001) with the solution obtained by the recursive inversion scheme of Rodgers (1976). These devices are not only valuable in terms of efficiency and flexibility for inverting model parameters such as $P$-wave velocity distribution, but particularly powerful if we attempt the practice of real-time seismic tomography in future, that is, we investigate temporal variations in model. In addition to the merits of the Huygens' method discussed in, for example, Saito (2001) and Rawlinson and Sambridge (2004), we can compute the ray path and travel time from every grid point in model to a given station together, by collocating source and station. We had better compute them before a new event takes place for the updated velocity model at hand obtained by the travel-time data for all the events in the past. We can revise the velocity model immediately after the occurrence of a new event because the required matrix element and data residual have been already prepared.

The recursive inverse scheme is particularly suitable for real-time seismic tomography because we only need simple vector-like computations such as Eqs. (12) and (13), if we like to add small amounts of new travel-time data. For example, suppose that we have already obtained the data in the past consisting of the total number of $N=10,000$ travel times. The computational time of our recursive inverse scheme is grossly similar or might be longer than that of some conventional inverse schemes such as iterative algorithms. On the other hand, we are supposed to obtain one travel-time observation for an event newly occurring in a studied area. We must solve the inverse problem of a large scale $(N=10,001)$, similar to the previous one, in the conventional seismic tomography. In contrast, our recursive inverse scheme only requires one additional computation for the $i=10,001$-th data set in Eqs. (12) and (13), which will be of the order of $1 / N$ times the original computational amount. That is, combining with the already-stored information of ray paths and travel times obtained by the Huygens' method a priori, we can quickly obtain a result
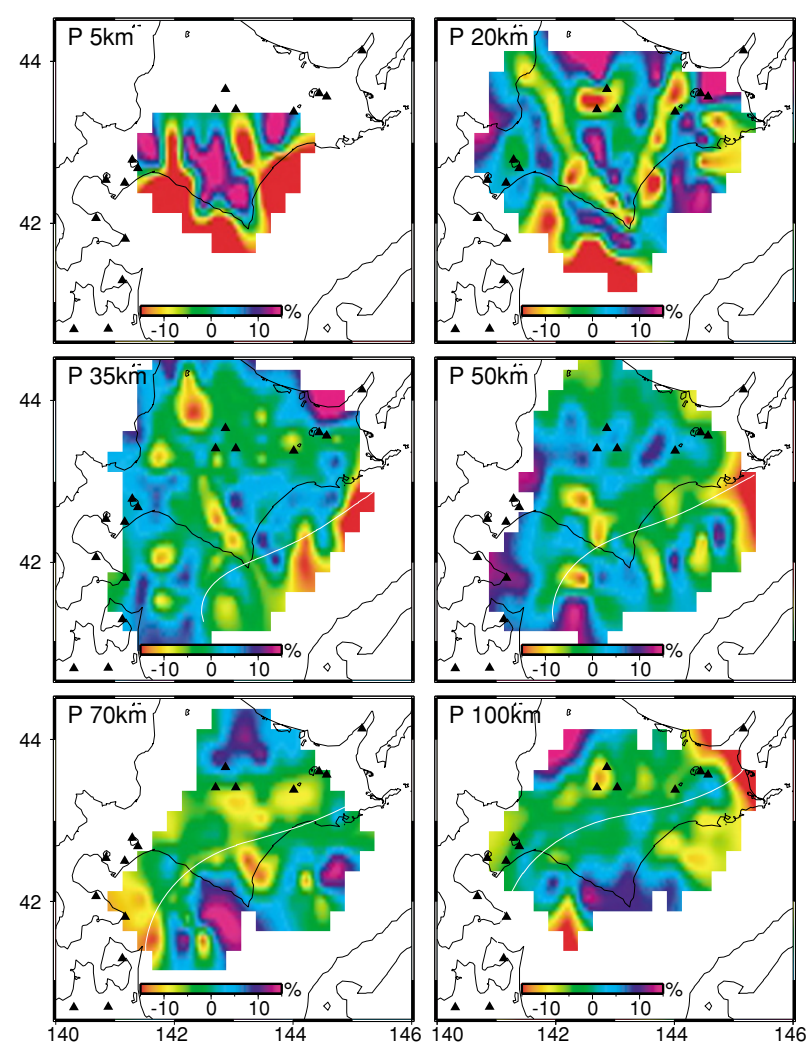

Fig. 11. $P$ wave velocity perturbations derived by Katsumata et al. (2003). Triangles indicate active volcanoes. (After Katsumata et al., 2003)

for the new entire data with $N=10,001$. We can also easily check how the result (i.e, the inverted velocity distribution) varies from $N=10,000$ to $N=10,001$, as its temporal variation.

In this study, we first investigated the stability and accuracy of the recursive inverse scheme for synthetic data with a given velocity model. The result obtained was compared with the one by a conventional iterative inverse scheme, confirming that our result agrees well with the conventional one. Moreover, the introduction of the upgraded reference velocity model at each recursive step enhances the spatial resolution of the final result in some cases. The use of the upgraded velocity model requires large computations for the conventional inverse scheme, so our new scheme rather improves final results in seismic tomography without computational cost.

In the end, we applied our recursive inverse scheme to the actual travel-time data in the Hidaka, Hokkaido, Japan, area, where a three-dimensional $P$-wave velocity structure of high resolution was already obtained by Katsumata et al. (2003). Our result accurately reproduced the result of Katsumata et al. (2003), showing the applicability of our method to travel-time data with realistic velocity models as well as actual source-station geometry in contemporary seismic tomography. In addition, the use of the upgraded reference velocity model in our method could retrieve some detailed velocity anomalies.

As mentioned in Introduction, real-time tomography has been operated in other branches of science and engineering, including a part of GPS observations in earth science. Since 
temporal variations are predicated to be very complex and subtle or only exist in a long term, very precise and reliable inverted results are required to detect them. Following conventional inverse schemes, we need to solve two independent inversion problems with two different data sets, for example, refer to a recent work by Foulger et al. (2003). Since we cannot avoid the introduction of subjective (a priori, in other words) parameters such as a timing of the convergence of iterations and a model covariance matrix, the temporal variation detected by this approach is extremely difficult to evaluate objectively with the present quality of data in earthquake seismology.

In contrast, our recursive inverse scheme modifies model parameters at each data set one by one, as seen in Eq. (12). That is, we can fix the above subjective or a priori parameters through the entire inversion process. Dividing the entire data set into two subsets, we also confirmed that the order of data does not affect the final result, as shown in Figs. 7 and 8 . The reliability of our approach is proved because the formulations, Eqs. (12) and (13), appear to be strongly controlled by the order of data, while conventional inverse schemes do not explicitly include the order in theory. This test visualizes the potential of the recursive scheme to detect temporal variations in model, for example, comparing the result only by the data from early events (the left of Fig. 7) with that by adding the data for events occurring later (the left of Fig. 8).

This study only discusses theoretical and numerical aspects of our proposed approach, and the quality of data is indeed extremely critical in practice if we seriously attempt to detect temporal variations in model. Nevertheless, it is very important for this study to establish an appropriate and reliable inverse scheme for real-time seismic tomography for the first time. We plan to apply the present method to our seismic monitoring system so that inverted temporal variations will be evaluated in a more objective manner than any previous attempts in earthquake seismology.

Acknowledgments. The authors especially thank Junji Koyama and Kazunori Yoshizawa for various suggestions. They appreciate suggestions about the interpretation of velocity models in the Hidaka area provided by Takeo Moriya. Comments by Noriko Tsumura, Robert L. Nowack and the editor (Eiichi Fukuyama) helped them to improve the original manuscript.

\section{References}

Aki, K., Overview, in Seismic Tomography: Theory and Practice, edited by H. M. Iyer and K. Hirahara, pp. 1-8, Chapman \& Hall, London, 1993.

Aki, K. and W. H. K. Lee, Determination of three-dimensional velocity anomalies under a seismic array using first $\mathrm{P}$ arrival times from local earthquakes: 1. A homogeneous initial model, J. Geophys. Res., 81, 4381-4399, 1976.

Aki, K. and P. G. Richards, Quantitative Seismology, vol. 2, pp. 641-719, W. H. Freeman and Comp., San Francisco, 1980.

Aki, K., A. Christofferson, and E. S. Husebye, Determination of the threedimensional seismic structure of the lithosphere, J. Geophys. Res., 82, 277-296, 1977.

Ding, Z., Y. Zhao, H. Ren, J. S. Nelson and Z. Chen, Real-time phaseresolved optical coherence tomography and optical Doppler tomography, Opt. Exp., 10, 236-245, 2002.

Foulger, G. R., B. R. Julian, A. M. Pitt, D. P. Hill, P. E. Malin, and E. Shalev, Three-dimensional crustal structure of Long Valley caldera, California, and evidence for the migration of $\mathrm{CO}_{2}$ under Mammoth Mountain, J. Geophys. Res., 108(B3), 2147, doi:10.1029/2000JB000041, 2003.
GPS Research Group, in The National Center for Atmospheric Research \& the UCAR Office of Programs, http://www.gst.ucar.edu/gpsrg/, 2004. Hofmann-Wellenhof, B., H. Lichtenegger, and J. Collins, Global Positioning System: Theory and Practice, 5th Rev., 383 pp., Springer Verlag, Wien, 2001.

Iwasaki, T., O. Ozel, T. Moriya, S. Sakai, S. Suzuki, G. Aoki, T. Maeda, and T. Iidaka, Lateral structural variations across a collision zone in central Hokkaido, Japan as revealed from seismic refraction profilings, Geophy. J. Int., 132, 435-457, 1998.

Iwasaki, T. and Research Group of HOKKAIDO TRANSECT, Crustal section across the Hidaka collision zone, Hokkaido, Japan as inferred from seismic refraction/reflection profiling, in Abst. XXIII Gen. Assem. Int. Union Geod. Geophys., SS03/07A/A02-007, 2003.

Iyer, H. M. and K. Hirahara (eds), Seismic Tomography: Theory and Practice, 842 pp., Chapman \& Hall, London, 1993.

Katsumata, K. et al. (64 authors), Distribution of Hypocenters and Focal Mechanisms in and around the Hidaka Arc-Arc Collision Zone Revealed by a Dense Temporary Seismic Network, Bull. Earthq. Res. Inst., 77, 199-223, 2002 (In Japanese with English abstract).

Katsumata, K., N. Wada, and M. Kasahara, Newly imaged shape of the deep seismic zone within the subducting Pacific plate beneath the Hokkaido corner, Japan-Kurile arc-arc junction, J. Geophys. Res., 108(B12), 2565, doi:10.1029/2002JB002175, 2003.

Miyamachi, H. and T. Moriya, Velocity structure beneath the Hidaka Mountains in Hokkaido, Japan, J. Phys. Earth, 32, 13-42, 1984.

Miyamachi, H. and T. Moriya, Velocity structure and aftershock distribution of the 1982 Urakawa-oki earthquake, J. Phys. Earth, 35, 309-326, 1987.

Moriya, T., H. Miyamachi, O. Ozel, N. Ozel, T. Iwasaki, and M. Kasahara, Collision structure and seismotectonics of the Hidaka Mountains, Hokkaido, Japan, Struc. Geol., 42, 15-30, 1997 (in Japanese with English abstract).

Moriya, T., H. Okada, T. Matsushima, S. Asano, T. Yoshi, and A. Ikami, Collision structure in the upper crust beneath the southwestern foot of the Hidaka Mountains, Hokkaido, Japan, as derived from explosion seismic observations, Tectonophys., 290, 181-196, 1998.

Murai, Y., S. Akiyama, K. Katsumata, T. Takanami, T. Yamashina, T. Watanabe, I. Cho, M. Tanaka, A. Kuwano, N. Wada, H. Shimamura, I. Furuya, D. Zhao, and R. Sanda, Delamination structure imaged in the source area of the 1982 Urakawa-oki earthquake, Geophys. Res. Lett., 30, 1490, doi:10.1029/2002GL016459, 2003.

Nishi, K., A three-dimensional robust seismic ray tracer for volcanic regions, Earth Planets Space, 53, 101-109, 2001.

Nishigami, K., Deep crustal heterogeneity along and around the San Andreas fault system in central California and its relation to the segmentation, J. Geophys. Res., 105, 7983-7998, 2000.

Niu, F., P. G. Silver, R. Nadaeu, and T. McEvilly, Stress-induced migration of seismic scatters associated with the 1993 Parkfield aseismic transient, Nature, 426, 544-548, 2003.

Nolet, G. (ed.), Seismic Tomography: With Applications in Global Seismology and Exploration Geophysics, Kluwer Academic Pub., 386 pp., 1987

Paige, C. and M. A. Saunders, LSQR: An algorithm for sparse linear equations and sparse least squares, Assoc. Comput. Mach. Trans. Math. Software, 8, 43-71, 1982.

Park, B. H., M. D. Pierce, B. Cense, and J. F. de Boer, Real-time multifunctional optical coherence tomography, Opt. Exp., 11, 782-793, 2003.

Qin, F., Y. Luo, K. Olsen, W. Cai, and G. T. Schuster, Finite-difference solution of the eikonal equation along expanding wavefronts, Geophysics, 57, 478-487, 1992.

Rawlinson, N. and M. Sambridge, Wavefront evolution in strongly heterogeneous layered media using the fast marching method, Geophys. J. Int., 156, 631-647, 2004.

Rodgers, C. D., Retrieval of atmospheric temperature and composition from remote measurements of thermal radiation, Rev. Geophys. Space Phys., 14, 609-624, 1976.

Saito, H., Seismic Traveltime Tomography for Shallow Subsurface Explorations, Ph.D. thesis, 126 pp., Hokkaido University, Sapporo, 2001.

Sen, M. and P. L. Stoffa, Global Optimization Methods in Geophysical Inversion, Elsevier, 281 pp., 1995.

Shearer, P. M., Introduction to Seismology, Cambridge Univ. Press, 260 pp., 1999.

Taira, T. and K. Yomogida, Imaging of three-dimensional small-scale heterogeneities in the Hidaka, Japan region: Coda spectral analysis, Geophys. J. Int., 158, 998-1008, 2004.

Takanami, T., Three-dimensional seismic structure of the crust and upper 
mantle beneath the orogenic belts in southern Hokkaido, Japan, J. Phys. Earth, 30, 87-104, 1982.

Tsumura, N., H. Ikawa, T. Ikawa, M. Shinohara, T. Ito, K. Arita, T. Moriya, G. Kimura, and T. Ikawa, Delamination-wedge structure beneath Hidaka collision zone, central Hokkaido, Japan inferred from seismic reflection profiling, Geophys. Res. Lett., 26, 1057-1060, 1999.

Vidale, J. E., Finite-difference calculation of traveltimes in three dimensions, Geophysics, 55, 521-526, 1990.

Wunsch, C., Using data with models: Ill-posed problems, in Oceanographic and Geophysical Tomography, edited by Y. Desaubies, A. Tarantola, and J. Zinn-Justin, pp. 203-248, North-Holland, Amsterdam, 1990.

Zeng, Y., Deterministic and stochastic modeling of the high-frequency
Seismic wave generation and propagation in the lithosphere, Ph.D. thesis, Univ. of South. Calif., Los Angeles, 1991.

Zhao, D., A. Hasegawa, and S. Horiuchi, Tomographic imaging of P and $\mathrm{S}$ wave velocity structure beneath northeastern Japan, J. Geophys. Res., 97, 19909-19928, 1992.

Zvyagin, A. V., J. B. FitzGerald, K. K. M. B. D. Silva, and D. D. Sampson, Real-time detection technique for Doppler optical coherence tomography, Opt. Lett., 25, 1645-1647, 2000.

M. Ogiso, K. Yomogida (e-mail: yomo@ep.sci.hokudai.ac.jp), and K. Katsumata 